\title{
Communication
}

Cite this: DOI:

$10.1039 / \times 0 \times x 00000 x$

\section{An Improved Transition-Metal-Free Synthesis of Aryl Alkynyl Sulfides via Substitution of a Halide at an sp- Centre.}

Roomi Mohima Chowdhury ${ }^{\dagger}$ and Jonathan D. Wilden ${ }^{\dagger *}$
Received 00 th January 2012, Accepted 00 th January 2012

DOI : $10.1039 / \times 0 \times x \odot \odot \odot \odot \odot x$

WwW.rsc.org/
A simple high-yielding preparation of aryl alkynyl sulfides is presented. The reaction of a chloroacetylene with a thiolate salt in the presence of an amine mediator (dimethylamine or $N, N^{\prime}$-dimethylethylenediamine) yields the alkynyl sulfides in excellent yields. The alkynyl chloride is easily prepared from the parent alkyne contrasting sharply with the cumbersome synthesis of an alkynyl sulfonamide previously required.

\section{Introduction}

Acetylinic sulfides ('thioynol ethers') are valuable synthetic intermediates with applications in a variety of processes. ${ }^{1}$ Their balance between stability and reactivity; being stable enough to purify and handle yet sufficiently reactive to undergo a wide variety of synthetic manipulations makes them (and their oxygen counterparts, ynol ethers) particularly versatile intermediates. ${ }^{2}$ The high electron density and polarity in the bond due to the resonance structures are outlined in figure 1:

$$
\mathrm{R}^{1} \stackrel{\curvearrowleft}{\Longleftarrow} \ddot{\mathrm{X}}^{2} \longleftrightarrow \stackrel{\mathrm{R}^{1}}{=} \stackrel{+}{\mathrm{X}}^{2} \quad \mathrm{R}^{1} \frac{\delta+}{\delta-} \ddot{\mathrm{X}}^{2}
$$

Figure 1. Polarity exhibited by acetylinic ethers and thioethers.

In particular, the high reactivity of the alkyne unit in cycloaddition processes is particularly valuable since complex molecules can be constructed in relatively few synthetic operations. Their reactions with electrophiles are similar to those of the related ynol ethers however the reactions of nucleophiles with these two classes of compounds are quite different. Ynol ethers tend to be attacked by nucleophiles at the $\alpha$-carbon atom (bearing the oxygen substituent) whereas thioynol ethers are usually attacked at the $\beta$-carbon atom. ${ }^{3}$ This property represents a potentially valuable 'Umpolung' strategy which has not yet been fully exploited.

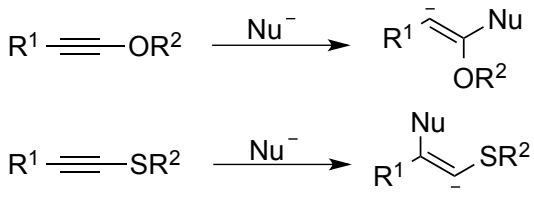

Scheme 1: Behaviour of acetylinic ethers and thioethers with nucleophiles.

Our previous work had established a synthesis of ynol ethers and thioynol ethers based on the displacement of a sulfonamide leaving group at the $s p$-centre of an aryl acetylene. ${ }^{4}$ Although this reaction works well, both in terms of reaction scope, rate and yield, the preparation of the alkynyl sulfonamide is non trivial and requires the preparation of the intermediate alkynyl sulfinamide followed by oxidation to the sulfonamide using $\mathrm{NaIO}_{4}$ with $\mathrm{RuCl}_{3}$ as a catalyst. ${ }^{5}$ Furthermore, the sulfonamide moiety as a leaving group represents poor atom economy and conflicts with our aspirations to undertake sustainable transformations (Scheme 2).

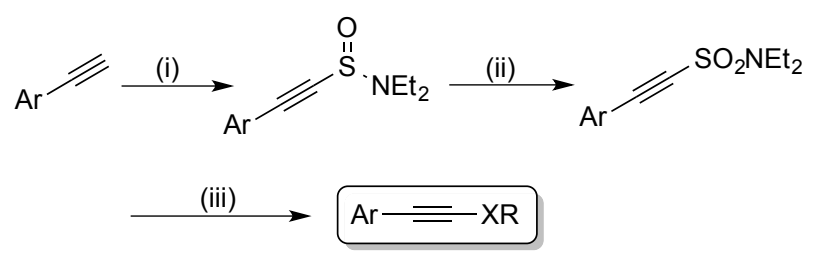

Scheme 2: Reagents and conditions: (i) $n \mathrm{BuLi}$ then $\mathrm{ClSONEt}_{2}$. (ii) $\mathrm{NaIO}_{4}, \mathrm{RuCl}_{3}$. (iii) $\mathrm{KXR}, \mathrm{THF}, 0^{\circ} \mathrm{C}, \mathrm{Me}_{2} \mathrm{NH}$.

\section{Results and Discussion}

We recognized that a halide would be a more atom efficient precursor and easier to prepare than the alkynyl sulfonamides since these can be prepared in a single step from the parent alkyne. As such we prepared chloro-, bromo- and iodophenylacetylene by known literature procedures (Scheme 3$){ }^{6}$ 


$$
\mathrm{Ar} \rightleftharpoons \frac{\mathrm{Ar}=\mathrm{Ph}}{\mathrm{Cl}, 80 \%} \mathrm{Ar}=\mathrm{X}
$$

Scheme 3: Reagents and conditions: $\mathrm{X}=\mathrm{Cl}: n \mathrm{BuLi}$ then NCS. $\mathrm{X}=$ Br: NBS, $\mathrm{AgNO}_{3}, \mathrm{Me}_{2} \mathrm{CO} . \mathrm{X}=\mathrm{I}: n$ BuLi then $\mathrm{I}_{2}$.

Exposure of each of these acetylinic halides to the potassium salt of $t$-butyl thiol under the conditions outlined in Scheme 2 gave the results shown in Scheme 4.

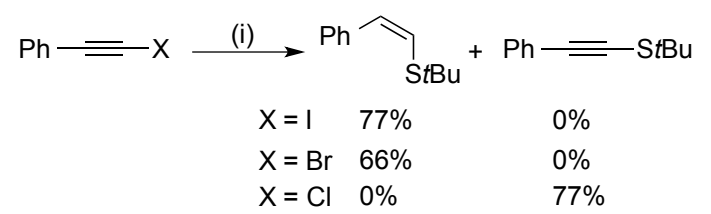

Scheme 4: Reagents and conditions: (i) $\mathrm{KSt} t \mathrm{Bu}, \mathrm{THF}, \mathrm{RT}$, DMEDA.

Pleasinglythe chloroacetylenes yielded the thioynol ether in good yield whereas both bromo and iodoacetylenes led to the thioenol ethers as shown in scheme 4. It appears that the weaker C-I and C-Br bonds allow a (well-documented) facile competing $\mathrm{X}$-philic reaction resulting in oxidation of the thiolate nucleophile. ${ }^{7}$ Protonation by trace amounts of moisture then lead to the parent alkyne that can then undergo addition reactions as we and others have previously described (Scheme 5$)^{8}$. The stronger $\mathrm{C}-\mathrm{Cl}$ bond is apparently able to resist the competing $\mathrm{X}$-philic pathway with the soft thiolate nucleophile and leads almost exclusively to the thioynol ether product in good yield.

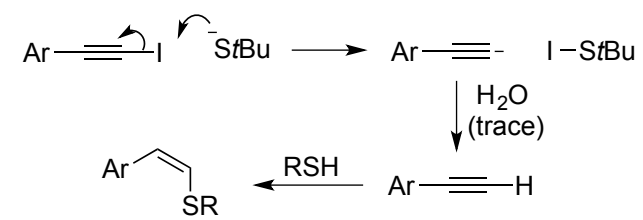

Scheme 5: X-philic reaction of thiolates with iodoacetylenes.

We also noted that if the reaction was performed in the presence of a small quantities of water $(2-5 \%)$ then the formation of the thioynol ether was greatly suppressed and the thioenol ether was isolated instead, predominantly as the $(Z)$-geometrical isomer, suggesting the involvement of a radical anion intermediate (Scheme 6). ${ }^{8}$ When water was replaced by $\mathrm{D}_{2} \mathrm{O}$, deuterium incorporation was observed in both vinylic positions. Presumably, when water is present, the hydroxide generated in the reaction medium undergoes the $\mathrm{X}$-philic reaction with the acetylinic chloride to yield the parent alkyne (phenylacetylene) which can then undergo addition of thiol as outlined in schemes 4 and 5 .

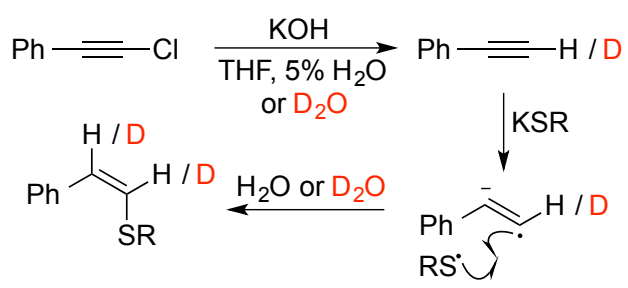

Scheme 6: Mechanistic pathway adopted when water is present.
The fact that alkynyl chlorides can be employed is significant since other methods of functionalizing acetylenes, particularly those that use transition metals, often rely on oxidative insertion into the weak $\mathrm{C}-\mathrm{X}$ bond and almost invariably this renders alkynyl chlorides unsuitable. For example earlier preparations of thioynol ethers from thiols and alkynyl iodides and bromides employs copper or palladium catalysis. ${ }^{9}$ The method outlined here therefore represents an alternative and potentially orthogonal method of preparing heteroatom substituted alkynes.

We then turned our attention to investigating the reaction scope. Initially we prepared a range of alkynyl chlorides from commercially available acetylenes by the method outlined in scheme 3 (1a-g, Figure 2). In general this preparation is uneventful however, $p$-bromophenylchloroacetylene, 1e, suffered a lower yield than the other examples, probably due to undesired metal-halogen exchange reactions when exposed to $n \mathrm{BuLi}$.

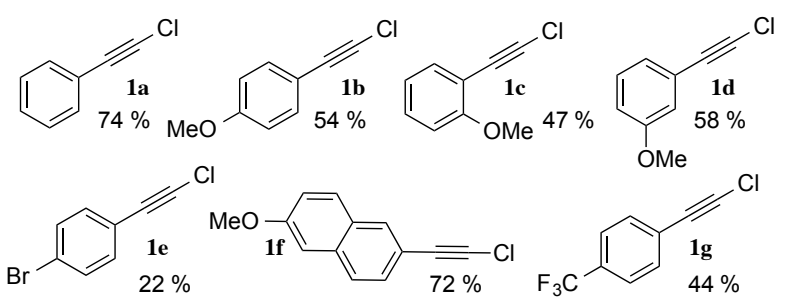

Figure 2: Range of chloroacetylenes 1a-g prepared.

Exposure of these acetylinic chlorides to the potassium salt of $t$-butyl thiol under the conditions shown in Figure 3 yielded the small library of acetylinic sulfides in excellent yields.

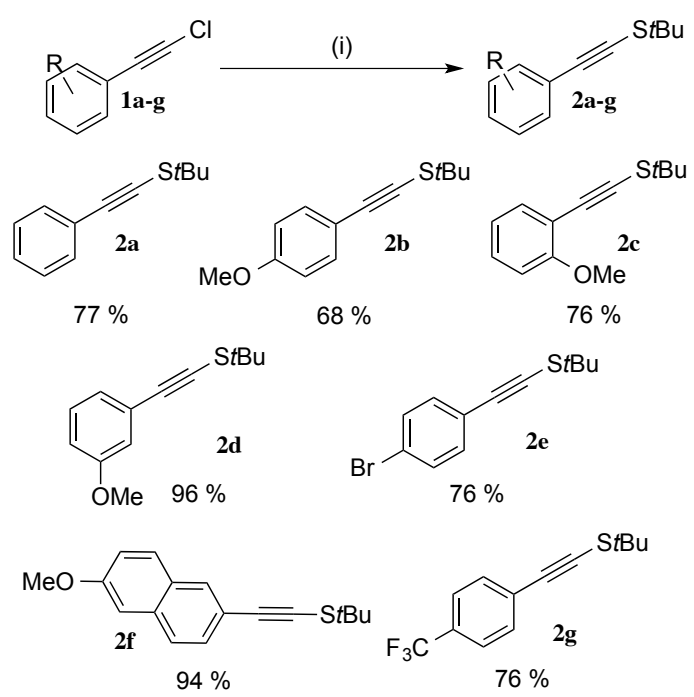

Figure 3: Reagents and conditions: (i) $\mathrm{KS} t \mathrm{Bu}, \mathrm{THF},-40-\mathrm{RT}$, $\mathrm{Me}_{2} \mathrm{NH}, 4 \mathrm{~h}$.

If the amine additive is removed from the reaction mixture the reaction still proceeds, however reaction times are significantly extended. The precise role of this additive and how it exerts its beneficial effect on the reaction is somewhat ambiguous. Although we and others have speculated as to possible mechanistic roles for these additives, a decisive conclusion cannot yet be drawn. It appears 
however that the additive may assist the initial electron transfer from the sulfur nucleophile to the alkyne. ${ }^{10,11}$ Mechanistically therefore, we postulate that the reaction proceeds through a similar pathway as for the displacement of the sulfonamide group to which we have dedicated considerable effort. ${ }^{5}$ This work has suggested that an addition-elimination mechanism is in operation but that radical and radical anion intermediates are involved (Scheme 7).

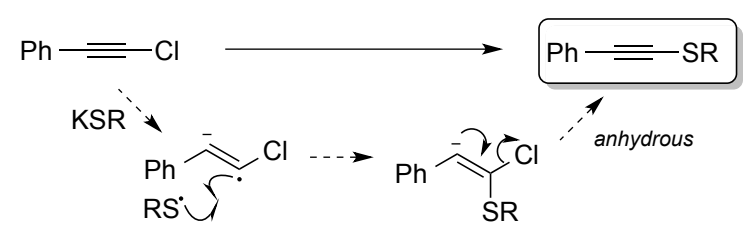

Scheme 7: Postulated reaction mechanism.

Finally, we have demonstrated that other sulfur nucleophiles can be employed. Substituting $t$-butyl thiolates with ethyl and $n$-hexyl analogues furnishes the corresponding ynol ethers in excellent yields (Figure 4).

$$
\mathrm{Ph} \underset{\mathbf{2 h} 64 \%}{\bar{\equiv}} \mathrm{S} \backsim \quad \mathrm{Ph} \underset{\mathbf{2 i} 69 \%}{=} \mathrm{S}\left(\mathrm{CH}_{2}\right)_{5} \mathrm{CH}_{3}
$$

Figure 4: Thioynol ethers bearing alternative R groups.

In conclusion a short and efficient approach to aryl thioynol ethers from the acetylinic chlorides has been described. These molecules have enormous synthetic potential and are difficult to prepare by other methods. No transition metals or heavy metal mediators are required and the use of chloride as the leaving group is more atom efficient and sustainable than other alternatives. Preliminary experiments suggest that a single electron transfer mechanism is in operation, which is consistent with our previous investigations in this field.

\section{Acknowledgments}

The authors gratefully acknowledge UCL for funding via the doctoral training centre in Drug Discovery.

\section{Notes and References}

$\dagger$ Department of Chemistry, University College London, 20 Gordon Street, London, WC1H 0AJ, UK. Email j.wilden@ucl.ac.uk

Electronic Supplementary Information (ESI) available: Full experimental detail, characterization data including ${ }^{1} \mathrm{H}$ and ${ }^{13} \mathrm{C} \mathrm{NMR}$ spectra are provided. See DOI: 10.1039/c000000x/

1 See for example (a) Hayashi, Y.; Narasaka, K. Chem. Lett. 1990, 1295-1298. (b) McConachie, L. K.; Schwan, A. L. Tetrahedron Lett. 2000, 41, 5637-5641.

2 See for example (a) Hilt, G.; Luers, S.; Harms, K. J. Org. Chem. 2004, 69, 624-630. (b) Spanka, C.; Schaumann, E. Synlett, 2014, 25, 2415-2428.

3 Radchenko, A. I.; Petrov, A. A. Russ. Chem. Rev. 1989, 948966.

4 Gray, V. J.; Slater, B.; Wilden, J. D. Chem. Eur. J. 2012, 18, 15582-15585.

5 Gray, V. J.; Cuthbertson, J.; Wilden, J. D. J. Org. Chem. 2014, $79,5869-5874$
6 Sud, D.; Wigglesworth, T. J.; Branda, N. R. Angew. Chem. Int. Ed. 2007, 8017-8019.

7 Zefirov, N. S.; Makhon'kov, D. I. Chem. Rev. 1982, 82, 615624.

8 (a) Tzalis, D.; Koradin, C.; Knochel, P. Tetrahedron Lett. 1999, 40, 6193-6195. (b) Bellucci, G.; Chiappe, C.; Lo Moro, G.; Synlett, 1996, 880-882. (c) Cuthbertson, J.; Wilden, J. D. Submitted to Tetrahedron.

9 (a) Brachet, E.; Brion, J-D.; Alami, M.; Messaoudi, S. Adv. Synth. Catal. 2013, 355, 2627-2636. (b) Braga, A. L.; Reckziegel, A.; Menezes, P. H.; Stefani, H. A. Tetrahedron Lett. 1993, 34, 393-394.

10 Cuthbertson, J.; Gray, V. J.; Wilden, J. D. Chem. Commun. 2014, 50, 2575-2578.

11 Zhou, S.; Doni, E.; Anderson, G. M.; Kane, R. G.; MacDougall, S. W.; Ironmonger, V. M.; Tuttle, T.; Murphy, J. A. J. Am. Chem. Soc. 2014, 136, 17818-17826. 